\title{
Rational control of structural off-state heterogeneity in a photoswitchable fluorescent protein provides switching contrast enhancement
}

\begin{abstract}
Authors: Virgile Adam ${ }^{1,4}$, Kyprianos Hadjidemetriou ${ }^{1,4}$, Nickels Jensen ${ }^{2,}$, Robert L. Shoeman ${ }^{3,4}$, Joyce Woodhouse', ${ }^{\prime,}$ Andrew Aquila ${ }^{4}$, Anne-Sophie Banneville', Thomas R. M. Barends ${ }^{3}$, Victor Bezchastnov $^{3}$, Sébastien Boutet ${ }^{4}$, Martin Byrdin', Marco Cammarata ${ }^{5}$, Sergio Carbajo ${ }^{4}$, Nina Eleni Christou', Nicolas Coquelle', Eugenio De la Mora', Mariam El Khatib', Tadeo Moreno Chicano', R. Bruce Doak $^{3}$, Franck Fieschi', Lutz Foucar', Oleksandr Glushonkov', Alexander Gorel $^{3}$, Marie Luise Grünbein ${ }^{3}$, Mario Hilpert ${ }^{3}$, Mark Hunter, Marco Kloos ${ }^{3}$, Jason E. Koglin', Thomas J. Lane ${ }^{4}$, Mengning Liang ${ }^{4}$, Angela Mantovanelli', Karol Nass ${ }^{3}$, Gabriela Nass Kovacs', Shigeki Owada ${ }^{6,7}$, Christopher M. Roome ${ }^{3}$, Giorgio Schirò', Matthew Seaberg ${ }^{4}$, Miriam Stricker ${ }^{3}$, Michel Thépaut ${ }^{1}$, Kensuke Tono ${ }^{6,7}$, Kiyoshi Ueda ${ }^{8}$, Lucas M. Uriarte', Daehyun You ${ }^{8}$, Ninon Zala', Tatiana Domratcheva ${ }^{10 *}$, Stefan Jakobs ${ }^{2}$, Michel Sliwa, Ilme Schlichting, JacquesPhilippe Colletier ${ }^{1}$, Dominique Bourgeois', Martin Weik ${ }^{1 *}$
\end{abstract}

\section{Affiliations:}

'Univ. Grenoble Alpes, CEA, CNRS, Institut de Biologie Structurale, F-38044 Grenoble, France

2Department of NanoBiophotonics, Max Planck Institute for Biophysical Chemistry, Göttingen, Germany and University Medical Center of Göttingen, Clinic for Neurology, Göttingen, Germany

${ }^{3}$ Max-Planck-Institut für medizinische Forschung, Jahnstrasse 29, 69120 Heidelberg, Germany

${ }^{4}$ Linac Coherent Light Source (LCLS), SLAC National Accelerator Laboratory, 2575, Sand Hill Road, Menlo Park, CA 94025, USA

'Department of Physics, UMR UR1-CNRS 6251, University of Rennes 1, Rennes, France. ${ }^{\circ}$ RIKEN SPring-8 Center, Sayo, Japan

Japan Synchrotron Radiation Research Institute, 1-1-1 Kouto, Sayo-cho, Sayo-gun, Hyogo 679-5198, Japan

${ }^{8}$ Institute of Multidisciplinary Research for Advanced Materials, Tohoku University, Sendai 980-8577, Japan

'Univ. Lille, CNRS, UMR 8516, LASIR, Laboratoire de Spectroscopie pour les Interactions, la Réactivité et l'Environnement, Lille 59000, France

${ }^{10}$ Department of Chemistry, Lomonosov Moscow State University, Moscow 119991, Russia

\# equal contribution

* correspondence: Tatjana.Domratcheva@mpimf-heidelberg.mpg.de,weik@ibs.fr 


\begin{abstract}
:
Reversibly photoswitchable fluorescent proteins are essential markers for advanced biological imaging, and optimization of their photophysical properties underlies improved performance and novel applications. Here we establish a link between photoswitching contrast, a key parameter that largely dictates the achievable resolution in nanoscopy applications, and chromophore conformation in the non-fluorescent state of rsEGFP2, a widely employed label in REversible Saturable OpticaL Fluorescence Transitions (RESOLFT) microscopy. Upon illumination, the cis chromophore of rsEGFP2 isomerizes to two distinct off-state conformations, trans1 and trans2, located on either side of the V151 side chain. Reducing or enlarging the side chain at this position (V151A and V151L variants) leads to single off-state conformations that exhibit higher and lower switching contrast, respectively, compared to the rsEGFP2 parent. The combination of structural information obtained by serial femtosecond crystallography with high-level quantum chemical calculations and with spectroscopic and photophysical data determined in vitro suggests that the changes in switching contrast arise from blue- and red-shifts of the absorption bands associated to trans 1 and trans 2 , respectively. Thus, due to elimination of trans2, the V151A variants of rsEGFP2 and its superfolding variant rsFolder2 display a more than two-fold higher switching contrast than their respective parent proteins, both in vitro and in E. coli cells. The application of the rsFolder2-V151A variant is demonstrated in RESOLFT nanoscopy. Our study rationalizes the connection between structural and photophysical chromophore properties and suggests a means to rationally improve fluorescent proteins for nanoscopy applications.
\end{abstract}

\title{
Introduction
}

Reversibly switchable fluorescent proteins (RSFPs $\left.{ }^{\prime \prime \prime}\right)$ are photochromic markers that are key to multiple super-resolution microscopy (nanoscopy) schemes including RESOLFT (Reversible Saturable Optical Fluorescence Transition ${ }^{21}$ ), NL-SIM (Non Linear Structured Illumination Microscopy ${ }^{(3)}$ ), SOFI (Super resolution Optical Fluctuation Imaging ${ }^{(4)}$ ) and multicolor PALM (Photo Activated Localization Microscopy ${ }^{(5)}$ ). They are also central tools in contrast enhancing approaches such as OLID (Optical Lock-In Detection microscopy ${ }^{\left({ }^{\circ}\right.}$ ) and in single channel multicolor approaches such as OPIOM (Out-of-Phase Imaging after Optical Modulation $\left.{ }^{[1]}\right)$. With the exception of Dreiklang ${ }^{[2 c \mid}$ and its variant SPOON ${ }^{[8]}$, RSFPs typically switch between a fluorescent on- and a non-fluorescent off-state through light-induced cis-trans isomerization of their p-hydroxybenzylidene imidazolinone chromophore ${ }^{[\mathrm{la}, 9]}$. Depending on 
whether the same wavelength that induces fluorescence switches the RSFP from the on- to the off-state or vice versa, they are said to be negative or positive, respectively ${ }^{[10]}$. At neutral $\mathrm{pH}$, the chromophore of negative RSFPs (such as rsEGFP2 and its rsFolder variants studied here) is generally cis-anionic in the on-state and trans-protonated in the off-state, although exceptions have been reported in rsGamillus "in. Off-switching is promoted by illumination with wavelengths near the fluorescence excitation maximum (typically $488 \mathrm{~nm}$ ) while $o n$-switching requires illumination in the near UV region (typically $405 \mathrm{~nm}$, Fig. 1a).

In all nanoscopy applications relying on RSFPs as labels, image quality and the achievable spatial resolution are mainly determined by the following photophysical characteristics ${ }^{[10]}$ : i) the fluorescence brightness, being defined as the product of the extinction coefficient of the $o n$-state and the fluorescence quantum yield, ii) the ensemble switching speed, i.e. the time required to switch the ensemble from the on- to the off-state, or vice versa, iii) the switching fatigue, being defined as the fraction of an RSFP ensemble being photobleached per full switching cycle, and iv) the switching contrast, that is, the ratio between the fluorescence signal after $o n$-switching and the residual signal after off-switching. Such residual fluorescence after off-switching mainly originates from back switching of the off-state chromophore by the off-switching light ${ }^{[12]}$. Among these characteristics, a high switching contrast is most critical for achieving high spatial resolution, and engineering efforts have thus been recently conducted to maximize it ${ }^{[1,133}$. If we neglect the possibility that the chromophore is not fully in the fluorescent cis-anionic state after on-switching ${ }^{[14 \mid}$, the switching contrast is a function of the on-to-off and off-to-on switching quantum yields and the extinction coefficients of both the on- and off-states at the off-switching wavelength (see eq. 1 in the Results section). In recent experimental ${ }^{\text {Iss }}$ and computational ${ }^{[1]}$ studies, it was proposed that the switching contrast is controlled by the relative stability of RSFPs in their on- and off-states via the number of hydrogen bonds between the chromophore and the protein pocket and its water molecules in each state. Here, focusing on rsEGFP2 and its rsFolder variants, we expand this view by showing how different conformations of the off-state chromophore modulate switching contrast, opening the door to rational optimization of RSFPs for enhanced nanoscopy applications.

rsEGFP2 (Figure 1a) has been generated based on EGFP and is widely applied in RESOLFT microscopy thanks to its fast maturation and favorable balance between fluorescence brightness, switching quantum yields, photofatigue resistance and switching contrast ${ }^{[1]}$. Recently, evidence for conformational heterogeneity in the off-state of rsEGFP2 at room temperature was provided by serial femtosecond crystallography (SFX), where in 
addition to the major trans state (trans 1 conformer), a hitherto unobserved trans isomer (trans 2 conformer) was observed ${ }^{[18]}$, which displays different twist and tilt dihedral angles $(\varphi$ and $\tau$ dihedral angles; see also Supplementary table S3), protein environment and H-bonding network (Supplementary figure S1). Interestingly, cryo-crystallographic synchrotron data revealed a similar trans2 isomer upon off-switching of an rsEGFP2 variant containing a monochlorinated chromophore when crystals with a contracted unit cell were examined, whereas a conformation similar to trans1 was populated in crystals with a larger unit cell ${ }^{[19}$. Trans1 and trans 2 conformers are located on either side of the V151 side chain (Supplementary figure S1), a residue that needs to transiently retract for the chromophore to switch between its cis and trans conformations (see supplementary movie in ${ }^{200}$ ) as suggested by time-resolved SFX ${ }^{[001}$. To start addressing the role of this residue in photoswitching, a variant with more space was generated by mutating V151 to an alanine ${ }^{2001}$. During the preliminary photophysical characterization ${ }^{1201}$ neither the switching contrast or the structure of the rsEGFP2-V151A variant was analyzed nor was the sEGFP2-V151L control with a more bulkier side chain generated.

The trans 2 conformation in rsEGFP2 is also very similar to the trans conformation observed in the off-state of rsFolder, a superfolding variant of rsEGFP2 that harbors a phenylalanine instead of a tyrosine at position 146 and that was designed to facilitate RESOLFT microscopy in "hostile" environments such as the bacterial periplasm " ${ }^{211}$. Strikingly, rsFolder has a much lower switching contrast than rsEGFP2 and rsFolder2, a single mutant of rsFolder with a tyrosine at position 146 and thus the same chromophore pocket as rsEGFP2 ${ }^{211}$. This observation suggests a possible link between the presence of trans 2 and a reduced switching contrast, raising the intriguing hypothesis that removal of the trans 2 fraction in rsEGFP2 could further enhance its switching contrast.

Here, we investigate the structural off-state heterogeneity in rsEGFP2 and eliminated it in V151A and V151L variants by shortening or lengthening the amino-acid side chain at position 151, respectively. The V151A variant exhibits only the trans 1 conformation and a substantially higher switching contrast compared to the rsEGFP2 parent, whereas the V151L variant shows only the trans 2 conformation and a lower contrast. The effects of the V151A and V151L mutations on the switching contrast are reproduced in rsFolder2. We show that changes in switching contrast between the investigated variants mainly result from differences in extinction coefficients of the corresponding off-states at the off-switching illumination wavelength. Furthermore, we postulate rapid exchange dynamics between trans1 and trans 2 in parental rsEGFP2 and in rsFolder2 and suggest that the achieved equilibrium depends on 
environmental factors, a notion we refer to as "photoswichting fragility". Finally, we show that the V151A variants maintain their gain in switching contrast in vivo and investigate the potential of rsFolder2-V151A for RESOLFT nanoscopy.

\section{Results}

Structural heterogeneity in the off-state of parental rsEGFP2

In order to corroborate the observation of a second trans isomer in parental rsEGFP2 ${ }^{[18]}$, a follow-up SFX experiment was carried out at the Linac Coherent Light Source (LCLS) using microcrystals of parental rsEGFP2 of the same crystal batch used earlier ${ }^{118,201}$. The on-state crystals were photoswitched by $488 \mathrm{~nm}$ light ${ }^{[2]}$ and the room-temperature (RT) structure of the resulting off-state solved at $1.7 \AA$ resolution (PDB entry 7O7U; see SI for details). Both trans 1 and trans 2 chromophore conformations are again present (Figure 1b, Supplementary figure S2). They agree well with those observed earlier ${ }^{1181}$, as well as with those observed in cryocrystallographic structures of an rsEGFP2 variant containing a monochlorinated chromophore

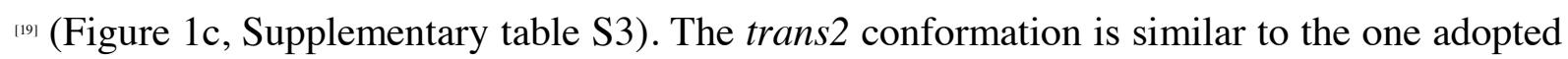
by rsFolder in its off-state ${ }^{211}$ (Figure 1d).

Structural heterogeneity bisected in the off-states of rsEGFP2 V151A and V151L variants

Given that the trans 1 and trans 2 chromophore conformations lie on either side of the V151 side chain (Figure 1b-d), we reasoned that this residue could also control the off-state heterogeneity. In addition to the rsEGFP2 variant with a shortened side chain (V151A $\left.{ }^{1001}\right)$, one with an enlarged (V151L) side chain was therefore generated and non-fluorescent off-state structures of both solved from RT SFX data collected at the SPring-8 Angstrom Compact free electron Laser (SACLA) from microcrystals after $488 \mathrm{~nm}$ light illumination ${ }^{221}$ (Figure 2, Supplementary figure S3, Supplementary table S2). The off-state structures (V151A: PDB entry 7O7X, V151L: PDB entry 7O7W) display only one chromophore conformation: trans1 for V151A (Figure 2a) and trans2 for V151L (Figure 2b). Absorption spectroscopy indicates that about $85 \%$ and $77 \%$ of microcrystalline rsEGFP2-V151A and -V151L chromophores have switched from the on- to the off-state, respectively (Supplementary figure S4). Trans1 and trans 2 chromophore conformations were modelled at $80 \%(75 \%)$ occupancy in rsEGFP2V151A (V151L) and the residual cis conformer at 20\% (25\%). Spectroscopic and crystallographic on- and off-state occupancies are thus consistent. In addition to differences in chromophore conformations, the off-states of the two variants also differ in their His149 and 
Tyr146 conformations. In the V151A variant His149 forms a hydrogen bond with Tyr146 and the trans 1 chromophore forms a hydrogen bond with a water molecule (distance: $2.7 \AA$, Supplementary figure S1c, Figure 2a), whereas in the V151L variant His149 is hydrogen bonded to the trans 2 chromophore (distance of $2.5 \AA$ between the chromophore phenol group and His149ND1; Supplementary figure S1b, Figure 2b). Synchrotron cryo-crystallography structures of rsEGFP2-V151A (off-state: PDB entry 7O7C , on-state: PDB entry 7O7D) and V151L (off-state: PDB entry 7O7H , on-state: PDB entry 7O7E) also feature a trans1 and trans2 chromophore in their off-state, respectively (Supplementary figure S5b, d) and a cis chromophore in the on-state (Supplementary figure S5a, c).

Overall, the RT SFX structures strongly suggest that the conformational off-state heterogeneity (trans1, trans2) seen in parental rsEGFP2 is eliminated in the rsEGFP2-V151A and -V151L variants, with trans 1 being occupied in the former and trans 2 in the latter. Thus, the residue at position 151 controls the off-state chromophore conformations (for a discussion of the modulation of conformational off-state heterogeneity see Supplementary Text S4).

Occupancies of trans1 and trans2 conformations in parental rsEGFP2 and rsFolder2 are sensitive to experimental conditions

A puzzling observation is that the relative occupancies of the trans1 and trans 2 conformations differ in off-state crystal structures of rsEGFP2 determined from three different SFX data sets, although the same batch of microcrystals was used (Supplementary text S1). In addition, attempts to observe the trans 2 conformation in macrocrystals of parental rsEGFP2 upon RT illumination at various intensities by synchrotron cryo-crystallography remained unsuccessful (see Supplementary text S2). In contrast, the rsFolder2 off-state structure determined by synchrotron cryo-crystallography (PDB entry 7AMF) showed residual occupancy of the trans 2 chromophore in addition to a mainly occupied trans 1 (Supplementary figure S6), similarly to the parental rsEGFP2 structures determined by RT SFX. These results suggest that relative occupancies of the two off-state conformations may change depending on even subtle differences in experimental conditions (see also $\left.{ }^{(19}\right)$. In contrast, the rsEGFP2V151A and -V151L structures in their off-states determined by SFX (Figure 2) are similar to those derived from cryo-crystallographic data we collected from macrocrystals at the European Synchrotron Radiation Facility (ESRF) (Supplementary table S1; Supplementary figure S5). 
Determination of switching contrast, switching quantum yields and extinction coefficients of rsEGFP2, rsFolder 2 and their variants embedded in polyacrylamide gels

To explore a possible correlation between the off-state chromophore conformations and the switching contrast, we measured the switching kinetics of rsEGFP2-V151A, rsEGFP2V151L and parental rsEGFP2 that contain either trans1, trans2, or both conformations, respectively. We also investigated rsFolder and rsFolder2, as well as the two variants rsFolder2V151A and rsFolder2-V151L.

We embedded the seven investigated variants in polyacrylamide gels and recorded their fluorescence switching curves under laser illumination at $488 \mathrm{~nm}$, using a wide field fluorescence microscope (Figure 3). The switching contrast was calculated as the ratio of the initial fluorescence in the $o n$-state after illumination at $405 \mathrm{~nm}$ divided by the residual steadystate fluorescence after off-switching with $488 \mathrm{~nm}$ light (Table 1). We found that for both rsEGFP2 and rsFolder2 the switching contrast is reduced $(\times \sim 0.33)$ in the V151L variants and increased $(x \sim 2.6)$ in the V151A variants compared to the parent proteins. The switching contrasts measured in the V151L variants $(\sim 15)$ are similar to that of rsFolder $(\sim 20)$, whereas that measured for both V151A variants exceeds 100 .

To explore the underlying reason for the modified switching contrasts, we examined the photoswitching kinetics of all variants in more detail. Neglecting the slow thermal relaxation in RSFPs ( $\sim$ hour range) in view of the timescale of our experiments ( $\sim$ second range), the switching contrast $S C$ at wavelength $\lambda$ can be approximated by:

$$
S C(\lambda) \simeq \frac{k_{\text {on } \rightarrow \text { off }}}{k_{\text {off } \rightarrow \text { on }}}=\frac{\varepsilon_{\lambda, \text { on }} \times \phi_{\text {on-to-off }}}{\varepsilon_{\lambda, \text { off }} \times \phi_{\text {off-to-on }}}
$$

where $k_{\text {on } \rightarrow \text { off }}$ and $k_{\text {off } \rightarrow \text { on }}$ are the on-to-off and off-to-on switching rates, $\phi_{\text {off-to-on }}$ and $\phi_{\text {off-to-on }}$ are the on-to-off and off-to-on switching quantum yields, respectively, and $\varepsilon_{\lambda \text {, on }}$ and $\varepsilon_{\lambda}$, off are the extinction coefficients of both the on and off states at the off-switching wavelength, respectively. Changes in the switching contrast can thus be due to changes in switching quantum yields ${ }^{[15]}$ and/or extinction coefficients of the on- and/or off-states. For the proteins in the present study, the off-switching wavelength is $488 \mathrm{~nm}$. Whereas $\varepsilon_{488, \text { on }}$ was measured using the Ward method ${ }^{1231} \phi_{\text {on-to-off, }} \phi_{\text {off-to-on, }} \varepsilon_{488, \text { off }}$ values were calculated by fitting the experimental fluorescence switching curves (Figure 3) with a kinetic model (see Supplementary methods section and Supplementary text S3). Overall, the dominating effect underlying variations between the observed switching contrasts in the studied variants follows from significant 
differences in off-to-on (rather than on-to-off ) switching brightness at $488 \mathrm{~nm}$ (Table 1): the V151A variants switch on significantly less efficiently at $488 \mathrm{~nm}$ than the parent proteins, while the V151L variants switch on much more efficiently. The observed differences in the off-to-on switching brightness are mainly due to differences in $\varepsilon_{488, \text { off }}$ (reduced $\sim 2.4$ fold in rsEGFP2V151A, and increased $~ 1.9$ fold in rsEGFP2-V151L compared to parental rsEGFP2) and only to a minor extend to differences in off-to-on switching quantum yields (Table 1). Note that absolute values of off-to-on switching quantum yields are notoriously difficult to determine, as evidenced by the spread in values determined in different laboratories (e.g. for rsEGFP2 in solution, values of $0.12^{[24 \mid}$ and $0.34^{[21}$ have been published). Yet, values determined under identical conditions in the same laboratory should be comparable. It is thus striking that similar off-to-on-switching quantum yields were measured here on rsEGFP2 (0.23) and rsEGFP2V151A (0.25) embedded in polyacrylamide gels, but a two-fold increase of the quantum yields for the proteins in solution has been reported by us earlier $(0.40$ and 0.77 for rsEGFP2 and rsEGFP2-V151A, respectively ${ }^{(20)}$ ). Unlike data in Table 1, the value for rsEGFP2-V151A in ${ }^{200}$ was determined from a single measurement and we suspect that an unidentified experimental flaw lead to the erroneously high value $(0.77)$ that should thus be discarded. Compared to rsEGFP2 and rsFolder2, the behavior of rsFolder is similar to that of the V151L variants. We note that the V151A variants are characterized by a lower fluorescence brightness than their parents (Table 1).

Overall, our results suggest that the higher and lower switching contrasts of the V151A and V151L variants relative to the parent proteins, respectively, are mainly due to lower absorptions of $488 \mathrm{~nm}$ light ( $\left.\varepsilon_{488, \text { off }}\right)$ by the trans1 chromophore in V151A and to higher absorption of the trans2 chromophore in V151L.

\section{$U V$-visible absorption spectroscopy on rsEGFP2, rsFolder 2 and their variants in solution}

The differences in $\varepsilon_{488, \text { off }}$ determined from fitting the experimental fluorescence switching curves (Table 1) can be rationalized by comparing UV-visible absorption spectra of rsEGFP2, rsFolder2 and their V151A and V151L variants in their on- and off-states, respectively (Figure 4). Indeed, we consistently observed that the maximum of the off-state spectra of the V151A variants are blue shifted, and the spectra of the V151L variants red shifted relative to those of the parent proteins (Table 1). In the V151A variants, the blue shifted off-state absorbance band leads to a lower residual extinction coefficient at $488 \mathrm{~nm}$ ( $\varepsilon 488$,off), resulting in less $o n$-state contamination after off-switching (Figure 4) and thus to an increased switching contrast. In 
contrast, the red shifted off-state absorbance band in the V151L variants leads to a higher extinction coefficient at $488 \mathrm{~nm}$, more on-state contamination and a lower contrast. Interestingly, a shoulder at $440 \mathrm{~nm}$ is visible in the off-state absorbance spectra of rsEGFP2V151L, rsFolder2-V151L and rsFolder (Figure 4) that cannot be attributed to residual absorbance of the on-state. This shoulder is also visible in off-state absorption spectra of microcrystalline rsEGFP2-V151L (Supplementary figure S4b).

We also recorded absorption spectra along on-to-off switching under alternating $488 \mathrm{~nm}$ illumination for all variants in the solution state. An isosbestic point (Supplementary figure S7) was always observed, suggesting a homogenous off-state, not only for the V151A and V151L variants, but also for the parent proteins. Likewise, all our fluorescent switching curves (Supplementary figure S8) displayed a similar trend, with no sign of more complex kinetics in the parent proteins compared to the V151A and V151L variants.

\section{Quantum chemical calculation analysis of chromophore conformations in parental rsEGFP2}

So far, we implicitly assumed that the trans 1 and trans 2 conformations observed in the crystal structures occur in the proteins in solution, and hence, the blue- and red-shifted absorption could be attributed to trans 1 and trans 2 chromophores, respectively. To test this assumption, the absorption spectra of the trans 1 and trans 2 conformers were characterized by quantum chemistry calculations. Starting from the SFX structure of parental rsEGFP2 (PDB entry 6T39 $\left.{ }^{(18)}\right)$, the geometries of models featuring trans1 and trans2 chromophore conformations in their respective protein environment (i.e. transl being H-bonded to a water molecule and trans2 to His149; Figure 5a) were optimized. The planarity of the chromophore remained similar to the experimentally derived one (Supplementary table S3) with trans 1 being rather distorted from the planar configuration in contrast to trans2 (Figure 5a). The two configurations also differ in the length of the phenolic $\mathrm{OH}$ bond and bonds of the conjugated system indicating a stronger binding of the phenolic proton concomitant with a reduced $\pi$ conjugation in trans 1 as compared to trans 2 . The energy cross sections computed for the phenolic $\mathrm{OH}$ bond stretching (Figure 5b) demonstrate a shape typical for the protonated phydroxybenzylidene imidazolinone chromophore interacting with a proton acceptor ${ }^{125]}$ and confirm a stronger proton binding in trans 1 . Consistent with reduced $\pi$-conjugation, the $S_{0}-S_{1}$ energy is higher for trans 1 than for trans2 (Figure 5b, Supplementary table S4), suggesting a blue-shifted absorption maximum for the former. The enhanced proton binging in trans 1 could be linked to a substantial electronic coupling of $15 \mathrm{meV}$ between trans 1 chromophore and 
electron donor Tyr146. The coupling is facilitated by a H-bond between His149 and Tyr146. The trans 2 chromophore is H-bonded with His149 itself, and its electronic coupling with Tyr146 is reduced to $0.5 \mathrm{meV}$. The absorption band shapes obtained with a quantummechanical model considering the one-dimensional $\mathrm{OH}$-stretching potential (Supplementary figure S9 and Supplementary table S5) also suggest a blue shifted absorption band of trans 1 compared to trans 2 .

Geometry optimization and excitation energy calculations were performed for the trans 1 and trans 2 models with alanine and leucine, respectively, at position 151. Similar distorted and planar geometries of trans1 and trans 2 , respectively, were found in these models (Supplementary figure S10 and Supplementary table S3). Further, the calculations confirmed that the $\mathrm{S}_{0}-\mathrm{S}_{1}$ excitation energy is higher for trans1 compared to trans 2 independent of the residue at position 151 (Supplementary table S4). Hence, our calculations suggest an increase of the excitation energy for trans 1 in comparison to trans2, consistent with the blue-shifted absorption band assigned to transl, which we correlate with the increased out of plane distortion and increased proton binding of trans1. The calculations thus corroborate our assumption that the trans 1 and trans 2 conformers are adopted both in crystallo and in solution.

Determination of in vivo switching contrasts of rsEGFP2, rsFolder2 and their variants and RESOLFT experiments on rsFolder2-V151A

To investigate the in vivo switching properties of $r s E G F P 2$, rsFolder2 and their variants at light intensities similar to those typically utilized in RESOLFT nanoscopy, we recorded switching curves on E. coli colonies expressing the respective proteins using high light intensities (Figure 6a, b). The determined contrasts of rsEGFP2-V151A (109) and rsFolder2V151A (119) increased and that of rsEGFP2-V151L (6) decreased with respect to parental rsEGFP2 (28) and rsFolder2 (22), in line with the results obtained with low light intensities in vitro (Table 1). We also compared the switching fatigue of parental rsEGFP2 and rsFolder2 and their variants. To this end, the fluorescence of E. coli colonies was switched on and off 4000 times and the maximal fluorescence of the on-state was recorded for every switching cycle (Figure 6c). While rsEGFP2 and rsFolder 2 could be switched more than 2000 times before their fluorescence was reduced to $50 \%$ of the initial brightness, the V151A variants could be switched $\sim 1500$ times. Based on in vivo switching properties, both V151A variants appeared to be suitable for RESOLFT nanoscopy. 
For investigating the usefulness for RESOLFT imaging, we decided to concentrate on rsFolder2-V151A, as this variant showed the highest photoswitching contrast in vitro (Table 1) and in vivo, and could be particularly useful because of its superfolding properties. A fusion protein of the cytoskeletal protein Keratin with rsFolder2-V151A was expressed in cultured human HeLa cells and imaged on the RESOLFT microscope. While the resulting image shows the expected clear improvement in resolution compared to the confocal counterpart (Figure 7), its resolution of $\sim 60 \mathrm{~nm}$ is comparable to the one of previous RESOLFT recordings on the same microscope using parental rsEGFP2 ${ }^{[20,}$, as judged by the full width at half maximum (FWHM) of line profiles across small Keratin-rsFolder2-V151A filaments. Hence, despite the increased switching contrast of the V151A variant, evidenced in vitro (Table 1) and in vivo (Figure 6), the recorded images do not show a significant increase in resolution compared to the parent protein. We mainly attribute this result to the lower molecular and cellular brightnesses of these variants compared to the parents (Table 1).

\section{Discussion}

\section{Correlation between off-state conformations, switching contrast and absorbance spectra}

We establish a link between the switching contrast and off-state occupancy of the trans 1 or trans 2 chromophore conformations in rsEGFP2 and rsFolder2 variants by combining our structural and photophysical results. For the trans 1 conformer (V151A variants), the switching contrast is high, whereas the trans 2 conformer (V151L variants) leads to a lower contrast. Parental rsEGFP2 and rsFolder2, exhibiting trans1/trans2 off-state heterogeneity (see Supplementary text S4), display an intermediate contrast (Table 1). The quantitative evaluation of fluorescence switching kinetics and the off-state absorbance spectra of the investigated variants strongly suggest that the main contribution to modulation of the switching contrast arises from red- and blue-shifted absorption maxima of the off-state in the V151L and V151A variants, respectively. This observation correlates with the differences in twist and tilt dihedral angles of the chromophore in trans 1 and trans 2 , as well as in its local environment, that tune the energy gap between the $\mathrm{S}_{0}$ and $\mathrm{S}_{1}$ electronic states as shown by our high-level quantum chemistry calculations. The red-shift of the experimental absorption maximum of the rsEGFP2V151L off-state was reproduced computationally (Figure 5b) and is consistent with the more extended electron delocalization in the near-planar chromophore of its trans 2 conformation compared to trans 1 (Figure 5a). The less planar trans 1 chromophore conformation might result 
from stabilization of the protonated form by charge transfer (CT) from Tyr146, activated by $\mathrm{H}-$ bonding to His149, that is absent for trans2.

When linking structural and spectroscopic results, we rely on the assumption that the off-state heterogeneity observed in crystals of parental rsEGFP2 and rsFolder2 is maintained in solution. Our high-level quantum chemistry calculations justify this assumption. Yet, the presence of isosbestic points in the absorbance spectra along off-switching (Supplementary figure S7) and detection of a single long-lived off-state in our fluorescence-based switching curves (Supplementary figure S11) indicate a homogeneous off-state in solution. These findings are consistent if we postulate a fast exchange between trans 1 and trans 2 in parental rsEGFP2 and rsFolder 2 in solution, occurring on timescales faster than detectable $(\sim 0.1 \mathrm{~s})$ in our absorbance and switching kinetics measurements. Our measurements in solution thus capture the average photophysical behavior between those of trans1 and trans2, giving rise to an apparently homogeneous off-state. The postulated fast exchange contrasts the assumption by Chang et al that trans conformations are locked on the second time scale (see Supplementary information $\mathrm{S} 3$ in $\left.{ }^{(19)}\right)$.

\section{Photoswitching fragility in parental rsEGFP2 and rsFolder2}

The differential trans2 occupancies found in off-state structures of rsEGFP2 determined by RT SFX under identical buffer conditions on microcrystals of the same batch but of different age and with varying illumination conditions (Supplementary text S1), as well as the absence of trans2 in flash-cooled parental rsEGFP2 macrocrystals (Supplementary figure S12) and its presence in rsFolder2 (Supplementary text S2, Supplementary figure S6), suggest that experimental and environmental parameters determine to which extent trans 2 gets populated in parental rsEGFP2 and rsFolder2. We suggest that this is the result of a low, environmentally dependent, barrier in the protein conformational energy landscape ${ }^{[27}$ separating access to, or exchange between, trans 1 and trans2, a notion that we refer to as "switching fragility". Yet, the experimental conditions that would reproducibly control heterogeneity in the off-states of rsEGFP2 and rsFolder2 have not been identified.

Photoswitching fragility in parental rsEGFP2 or rsFolder2 may have consequences for imaging applications, when labels are addressed to various cellular locations with potentially different physicochemical environments. These different environments (e.g. viscosity, ionic strength, or nature of a fusion protein) could lead to different levels of heterogeneity and variability in switching contrast (beyond that expected from $\mathrm{pH}$ induced effects ${ }^{(11)}$ ). Such 
variability is expected to be alleviated in rsFolder, the V151L variants, and the high switching contrast V151A variants so that they may be considered as more "robust" than their parents.

In conclusion, this work establishes a causal relationship between the occupancy of two off conformations in rsEGFP2 and rsFolder2 and the achievable switching contrast, essentially through absorbance shifts of the off-switched chromophore. A point mutation is sufficient to enforce single trans conformations in the off-state. The rsEGFP2- and rsFolder2-V151A variants, containing only the trans 1 conformer, exhibit greatly enhanced switching contrasts as compared to their parents, both in vitro and in vivo. Due to a loss in fluorescence brightness of these variants, however, the optical resolution obtained by RESOLFT nanoscopy on KeratinrsFolder2-V151A filaments did not significantly increase compared to previous studies using either parental rsFolder2 or rsEGFP2. Our rsEGPF2-V151A and rsFolder2-V151A variants constitute promising leads for the next-generation RSFPs, for which the fluorescence brightness has to be increased while maintaining the enhanced switching contrast described here.

\section{Acknowledgment:}

We thank Elke De Zitter for critically commenting on the manuscript. The XFEL experiments were carried out at BL2-EH3 of SACLA with the approval of the Japan Synchrotron Radiation Research Institute (JASRI; Proposal No. 2018A8026; 27 - 29 July 2018) and at the CXI beamline at the LCLS (Proposal No. LM47 (23 - 27 June 2016) and LR38 (22 - 26 February 2018). We warmly thank the SACLA and LCLS staff for assistance. Use of the LCLS, SLAC National Accelerator Laboratory, is supported by the U.S. Department of Energy, Office of Science, Office of Basic Energy Sciences under Contract no. DE-AC02-76SF00515. Part of the sample injector used at LCLS for this research was funded by the National Institutes of Health, P41GM103393, formerly P41RR001209. We acknowledge support from the Max Planck Society. The study was supported by travel grants from the CNRS (GoToXFEL) to MW, an ANR grant to MW, MC, MS1 (BioXFEL), an ANR grant to DB (grant no. ANR-17-CE11-004701), a PhD fellowship from Lille University to LMU and an MENESR - Univ. Grenoble Alpes fellowship to KH, a Russian Science Foundation grant (project \#17-13-01051) to TD. This work was partially carried out at the platforms of the Grenoble Instruct-ERIC center (IBS and ISBG; UMS 3518 CNRS-CEA-UGA-EMBL) within the Grenoble Partnership for Structural Biology (PSB). Platform access was supported by FRISBI (ANR-10-INBS-05-02) and GRAL, a project of the University Grenoble Alpes graduate school (Ecoles Universitaires de Recherche) CBH- 
bioRxiv preprint doi: https://doi.org/10.1101/2021.11.05.462999; this version posted November 5, 2021. The copyright holder for this

preprint (which was not certified by peer review) is the author/funder. All rights reserved. No reuse allowed without permission.

EUR-GS (ANR-17-EURE-0003). The IBS acknowledges integration into the Interdisciplinary Research Institute of Grenoble (IRIG, CEA). 


\section{Main text Figures and Tables}

Table 1. Photophysical parameters obtained in vitro

\begin{tabular}{|c|c|c|c|c|c|c|c|}
\hline & rsEGFP2 & rsEGFP2-V151A & rsEGFP2-V151L & rsFolder2 & rsFolder2-V151A & rsFolder2-V151L & rsFolder \\
\hline Absorption max. anionic (on) [nm] & 482 & 483 & 483 & 483 & 484 & 483 & 479 \\
\hline Absorption max. neutral (off) $[\mathrm{nm}]$ & 403 & 397 & 405 & 397 & 397 & 400 & 402 \\
\hline Absorption max. switched (off) [nm] & 409 & 406 & 417 & 413 & 410 & 418 & 413 \\
\hline on-to-off-switching brightness $* \mathcal{\varepsilon}\left[\mathrm{M}^{-1} \mathrm{~cm}^{-1}\right]$ & $563 \pm 13$ & $673 \pm 9$ & $415 \pm 18$ & $690 \pm 28$ & $827 \pm 48$ & $455 \pm 15$ & $441 \pm 13$ \\
\hline$o n$-to-off-switching quantum yield, $\phi_{\text {on-to-off }}$ & $9.3 \times 10^{-3} \pm 0.2 \times 10^{-3}$ & $11.4 \times 10^{-3} \pm 0.2 \times 10^{-3}$ & $6.5 \times 10^{-3} \pm 0.3 \times 10^{-3}$ & $10.0 \times 10^{-3} \pm 0.4 \times 10^{-3}$ & $14.1 \times 10^{-3} \pm 0.8 \times 10^{-3}$ & $6.5 \times 10^{-3} \pm 0.2 \times 10^{-3}$ & $7.3 \times 10^{-3} \pm 0.2 \times 10^{-3}$ \\
\hline off-to-on-switching brightness ${ }^{\S}\left[\mathrm{M}^{-1} \mathrm{~cm}^{-1}\right]$ & $5807 \pm 233$ & $6590 \pm 134$ & $8713 \pm 193$ & $6054 \pm 173$ & $6852 \pm 198$ & $8979 \pm 210$ & $7877 \pm 125$ \\
\hline off-to-on -switching brightness ${ }^{\mathrm{S}}\left[\mathrm{M}^{-1} \mathrm{~cm}^{-1}\right]$ & $14.9 \pm 0.9$ & $6.8 \pm 0.8$ & $35.4 \pm 1.2$ & $16.9 \pm 1.1$ & $7.4 \pm 0.5$ & $39.3 \pm 1.7$ & $26.0 \pm 0.8$ \\
\hline off-to-on switching quantum yield, $\phi_{\text {off-to-on }}$ & $2.3 \times 10^{-1} \pm 0.09 \times 10^{-1}$ & $2.5 \times 10^{-1} \pm 0.05 \times 10^{-1}$ & $2.9 \times 10^{-1} \pm 0.06 \times 10^{-1}$ & $1.5 \times 10^{-1} \pm 0.04 \times 10^{-1}$ & $2.2 \times 10^{-1} \pm 0.06 \times 10^{-1}$ & $2.7 \times 10^{-1} \pm 0.06 \times 10^{-1}$ & $2.4 \times 10^{-1} \pm 0.04 \times 10^{-1}$ \\
\hline$\varepsilon_{\max , \text { on }}\left[\mathrm{M}^{-1} \mathrm{~cm}^{-1}\right]$ & 65474 & 62284 & 68295 & 72675 & 61091 & 74470 & 71871 \\
\hline$\varepsilon_{488, \text { on }}\left[\mathrm{M}^{-1} \mathrm{~cm}^{-1}\right]$ & 60807 & 59090 & 63851 & 69124 & 58798 & 70254 & 59509 \\
\hline$\varepsilon_{488, \text { off }}\left[\mathrm{M}^{-1} \mathrm{~cm}^{-1}\right]$ & $65 \pm 3$ & $27 \pm 3$ & $122 \pm 3$ & $111 \pm 6$ & $34 \pm 2$ & $145 \pm 5$ & $110 \pm 3$ \\
\hline$\varepsilon_{\max , 0 \text { fff }}\left[\mathrm{M}^{-1} \mathrm{~cm}^{-1}\right]$ & 25758 & 26694 & 33333 & 42434 & 32267 & 37079 & 38518 \\
\hline$\varepsilon_{405, \text { off }}\left[\mathrm{M}^{-1} \mathrm{~cm}^{-1}\right]$ & 25349 & 26670 & 29987 & 39841 & 31473 & 33061 & 36137 \\
\hline off-to-on thermal recovery time [hours] & 4.5 & 65 & 10 & 25 & 75 & 13 & 69 \\
\hline Switching contrast [fold-change] & $43 \pm 5$ & $119 \pm 21$ & $15 \pm 1$ & $46 \pm 3$ & $125 \pm 11$ & $15 \pm 1$ & $20 \pm 1$ \\
\hline Fluorescence brightness & 20952 & 16194 & 19123 & 24709 & 12829 & 33511 & 21561 \\
\hline Fluorescence quantum yield & $0.32 \pm 0.02$ & $0.26 \pm 0.01$ & $0.28 \pm 0.01$ & $0.34 \pm 0.01$ & $0.21 \pm 0.01$ & $0.45 \pm 0.04$ & $0.30 \pm 0.01$ \\
\hline Maturation time [min] & $39.1 \pm 4.2$ & $116.7 \pm 3.3$ & $90.8 \pm 7.7$ & $70.8 \pm 6.6$ & $157.7 \pm 2.3$ & $119.7 \pm 60.3$ & $77.2 \pm 5.3$ \\
\hline $\mathrm{pKa}$ & 5.9 & 6.4 & 5.7 & 5.8 & 6.4 & 5.9 & \\
\hline
\end{tabular}

*: Switching brightness refers to the product (switching quantum yield $\times$ extinction coefficient)

: Off switching brightness at $488 \mathrm{~nm}$

: On switching brightness at $405 \mathrm{~nm}$

: On switching brightness at $488 \mathrm{~nm}$

All parameters were determined by measurements on protein in solution at $\mathrm{pH} 7.5$ except switching brightness, switching quantum yield and switching contrast values that were extracted from measurements on protein

in polyacrylamide gel at $\mathrm{pH}$ 8.0. Values obtained from at least three independent measurements for each variant.

ND: Not Determined 

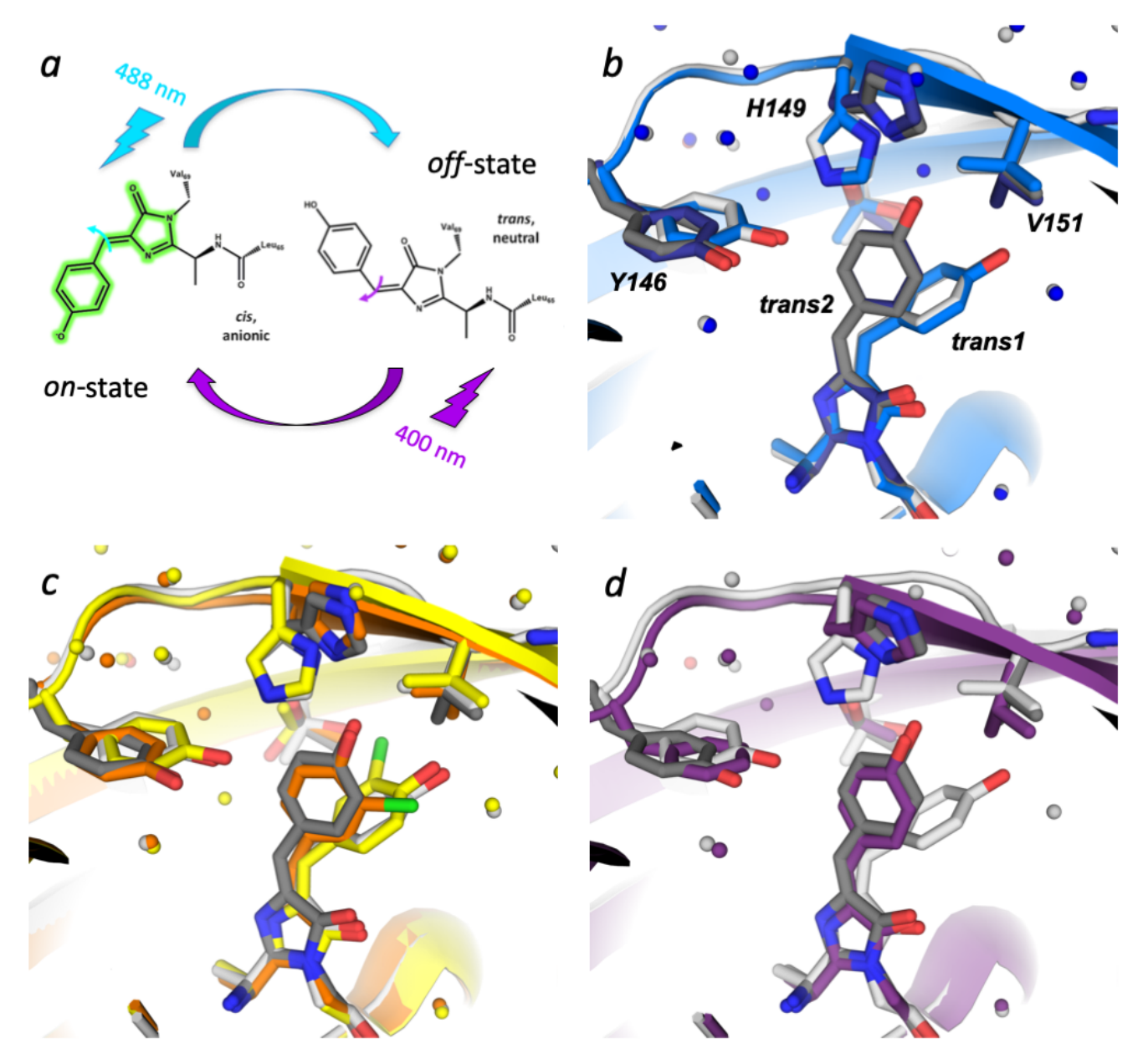

Figure 1: Photoswitching and off-state conformations in parental rsEGFP2. (a) rsEGFP2 can be photoswitched from the fluorescent on-state (anionic cis chromophore) to the nonfluorescent off-state (neutral trans chromophore) by illumination with $488 \mathrm{~nm}$ light, and back by illumination with 405-nm light. Photoswitching involves chromophore isomerization (blue and purple arrows on chromophore methylene bridge) and a change in protonation state of the phenol group. (b) Structures of parental rsEGFP2 in its off-state solved from RT SFX data. Offstate models of parental rsEGFP2 solved from RT SFX data earlier (PDB entry 6T39 ${ }^{18}$ ) and in this work (PDB entry 7O7U). Trans1 and trans2 are occupied at 40\% (light grey) and 30\% (dark grey) in parental rsEGFP2 (this work) and at 65\% (light blue) and 25\% (dark blue) in 6T39, respectively. $(c, d)$ Off-state models of parental rsEGFP2 solved from RT SFX data in this work (light and dark grey), overlaid with trans conformations in synchrotron structures of rsEGFP2 containing a monochlorinated chromophore ${ }^{[191}$ solved from crystals with looser (yellow) and tighter (orange) crystal packings ${ }^{[19 !}(c)$ and with the off-state model of rsFolder (PDB entry 5DU0 $\left.{ }^{211}\right)$ in purple $(d)$. 

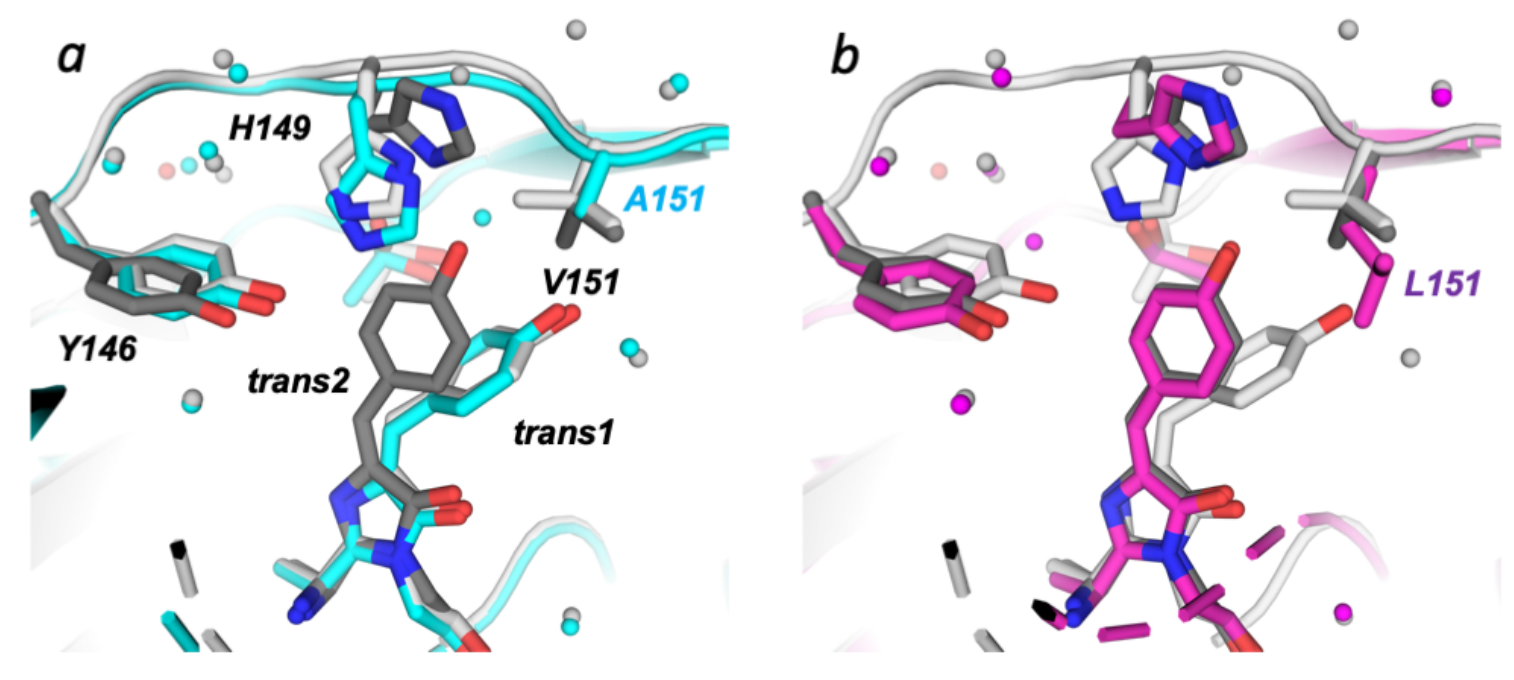

Figure 2: Structures of rsEGFP2 and its V151A and V151L variants in their off-states solved from RT SFX data. Off-state models of (a) rsEGFP2-V151A (cyan; PDB entry 7O7X) and (b) -V151L (purple; PDB entry 7O7W) variants are superimposed on the model of parental rsEGFP2 in the off-state solved from RT SFX data (PDB entry 7O7U), featuring trans1 in light grey and trans 2 in dark grey. Trans 1 and trans 2 are occupied at $80 \%$ and $75 \%$ in rsEGFP2 V151A and V151L, respectively. The cis conformers were removed for clarity. 
a

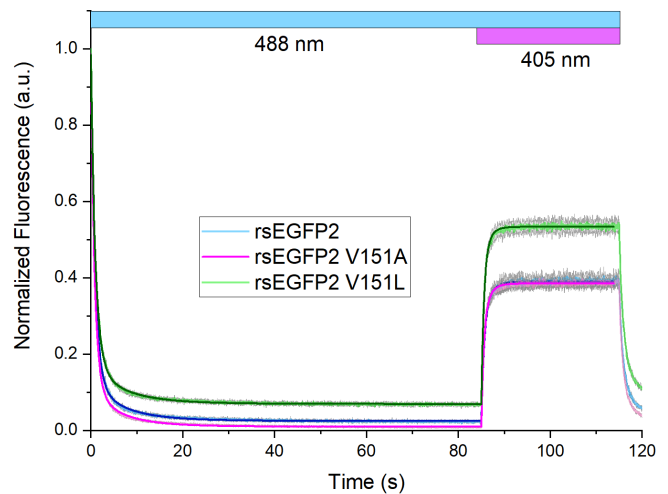

C

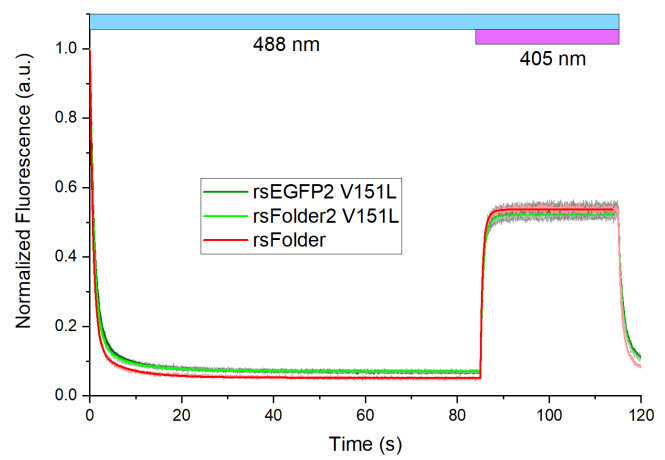

b

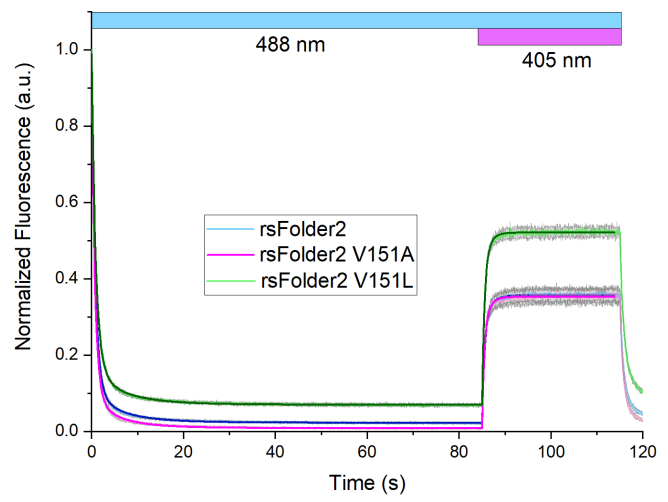

Figure 3: Fluorescence switching curves. $(a, b)$ Fluorescence switching curves for rsEGFP2 and rsFolder2 proteins together with their V151A and V151L variants. (c) Switching curve for rsFolder compared to V151L variants of rsEGFP2 and rsFolder2. Data obtained from in-vitro measurements of purified proteins, embedded in polyacrylamide gel $(\mathrm{pH} \mathrm{8.0)}$, on an epifluorescence microscope using $488 \mathrm{~nm}\left(0.27 \mathrm{~W} / \mathrm{cm}^{2}\right)$ illumination throughout data acquisition, and additional $405-\mathrm{nm}\left(0.03 \mathrm{~W} / \mathrm{cm}^{2}\right)$ during off-to-on switching. Pale colors stand for the mean values calculated from six measurements with standard deviations shown in grey, whereas dark solid lines represent the fits from the used kinetic model. 

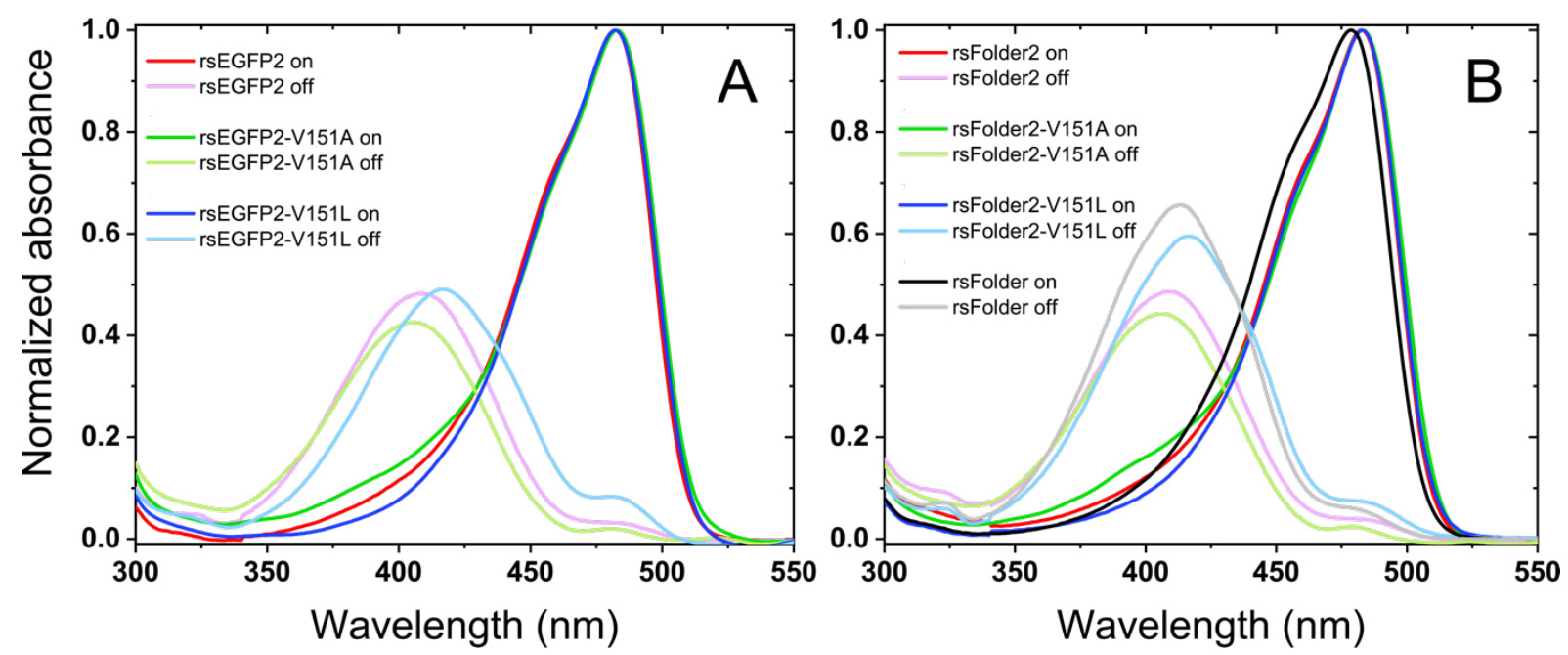

Figure 4: Absorption spectra of fluorescent proteins presented in this study in their fluorescent on-state (dark colors) and non-fluorescent off-state (dim colors) in solution. (a) rsEGFP2 and its variants rsEGFP2-V151A and rsEGFP2-V151L, (b) rsFolder2 and its variants rsFolder2-V151A and rsFolder2-V151L and rsFolder. Spectra are normalized relatively to the respective on state spectra and are measured in HEPES buffer at $\mathrm{pH}$ 7.5. 
a

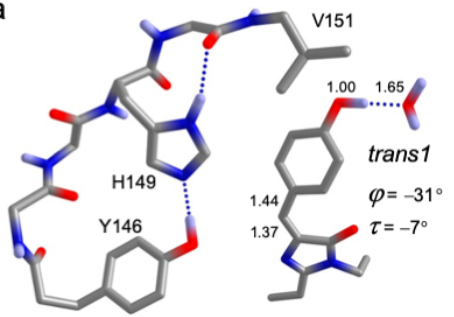

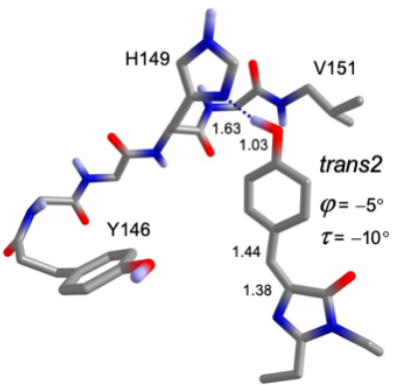

b

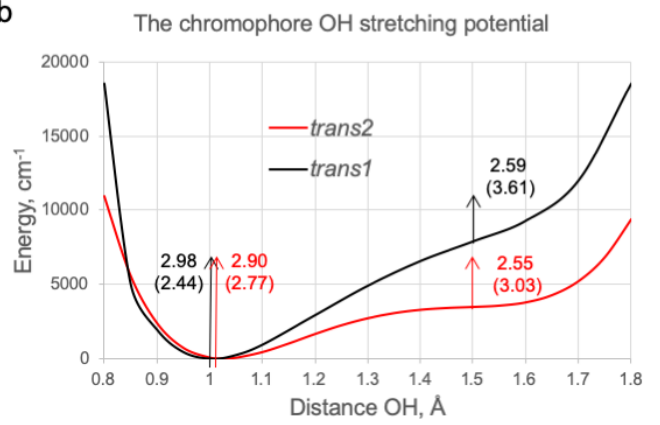

Figure 5: Quantum chemical calculations of trans1 and trans2 within parental rsEGFP2. (a) Fragments of the chromopore models and selected distances $(\AA)$ and angles in the optimized geometries. (b) The $\mathrm{OH}$ stretching energies. Vertical arrows indicate the $\mathrm{S}_{0}-\mathrm{S}_{1}$ excitation energies $(\mathrm{eV})$ and transition dipole moments (in brackets; Hartree*Bohr). The decrease of the $\mathrm{S}_{0}-\mathrm{S}_{1}$ energy along the $\mathrm{OH}$ stretching coordinate is demonstrated. The effect of the $\mathrm{OH}-$ stretching potentials on the $S_{0}-S_{1}$ vibronic band structure is presented in Supplementary figure S9. 

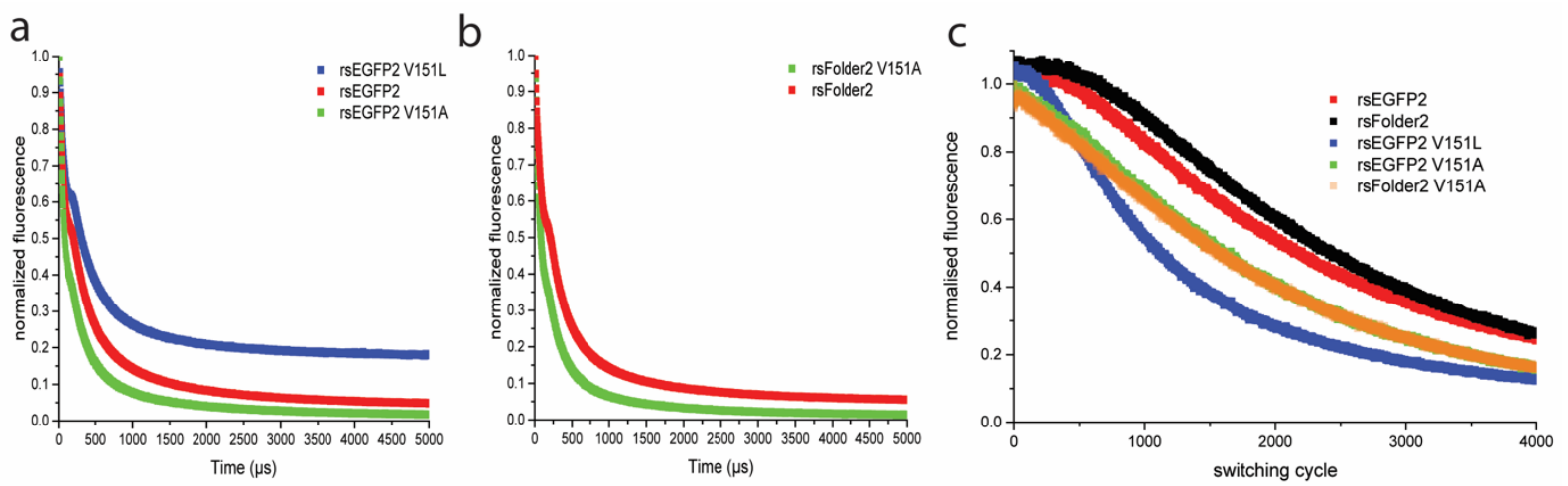

Figure 6: Switching kinetics and switching fatigue of rsEGFP2, rsEGFP2 V151A, rsEGFP2 V151L, rsFolder2 and rsFolder2 V151A. Comparison of the off switching curve of rsEGFP2, rsEGFP2-V151A and rsEGFP2-V151L $(a)$, as well as rsFolder2 and rsFolder2V151A (b). Switching fatigue measurements of the five proteins (c). All graphs were recorded on living $E$. coli colonies using high light intensities. 

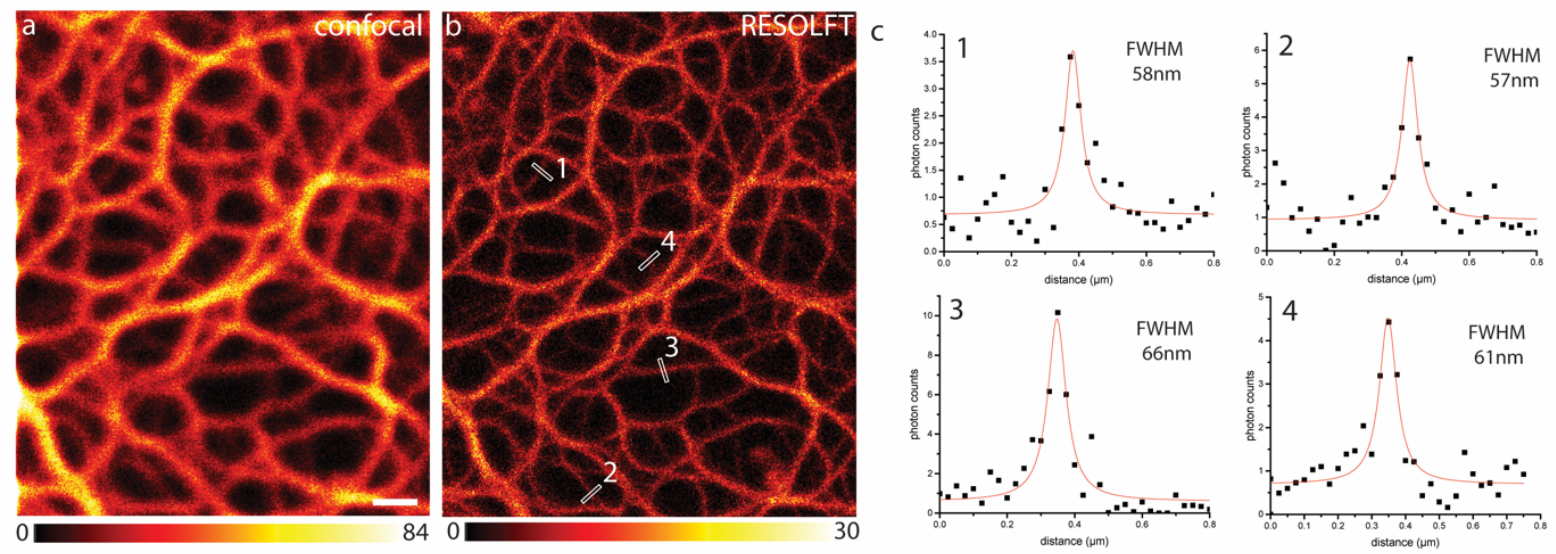

Figure 7: RESOLFT imaging using rsFolder2-V151A. Comparison of a confocal $(a)$ and RESOLFT $(b)$ recording of a HeLa cell expressing Keratin-rsFolder2-V151A. Line profiles were taken at the indicated positions and the FWHM was determined on the fitted functions (c). Scale bar: $1 \mu \mathrm{m}$. The color maps indicate the actual photon counts. 


\section{References}

[1] aD. Bourgeois, V. Adam, IUBMB Life 2012, 64, 482-491; bK. Nienhaus, G. U. Nienhaus, ACS nano 2016.

[2] aM. Hofmann, C. Eggeling, S. Jakobs, S. W. Hell, Proceedings of the National Academy of Sciences of the United States of America 2005, 102, 17565-17569; bT. Grotjohann, I. Testa, M. Leutenegger, H. Bock, N. T. Urban, F. Lavoie-Cardinal, K. I. Willig, C. Eggeling, S. Jakobs, S. W. Hell, Nature 2011, 478, 204-208; cT. Brakemann, A. C. Stiel, G. Weber, M. Andresen, I. Testa, T. Grotjohann, M. Leutenegger, U. Plessmann, H. Urlaub, C. Eggeling, M. C. Wahl, S. W. Hell, S. Jakobs, Nature Biotechnology 2011, 29, 942-947.

[3] M. G. L. Gustafsson, Proceedings of the National Academy of Sciences of the United States of America 2005, 102, 13081-13086.

[4] T. Dertinger, R. Colyer, G. Iyer, S. Weiss, J. Enderlein, Proceedings of the National Academy of Sciences 2009, 106, 22287.

[5] H. Shroff, C. G. Galbraith, J. A. Galbraith, H. White, J. Gillette, S. Olenych, M. W. Davidson, E. Betzig, Proceedings of the National Academy of Sciences 2007, 104, 20308.

[6] G. Marriott, S. Mao, T. Sakata, J. Ran, D. K. Jackson, C. Petchprayoon, T. J. Gomez, E. Warp, O. Tulyathan, H. L. Aaron, E. Y. Isacoff, Y. Yan, Proceedings of the National Academy of Sciences of the United States of America 2008, 105, 17789-17794.

[7] J. Quérard, R. Zhang, Z. Kelemen, M.-A. Plamont, X. Xie, R. Chouket, I. Roemgens, Y. Korepina, S. Albright, E. Ipendey, M. Volovitch, H. L. Sladitschek, P. Neveu, L. Gissot, A. Gautier, J.-D. Faure, V. Croquette, T. Le Saux, L. Jullien, Nature Communications 2017, 8, 969.

[8] Y. Arai, H. Takauchi, Y. Ogami, S. Fujiwara, M. Nakano, T. Matsuda, T. Nagai, ACS Chem Biol 2018, 13, 1938-1943.

[9] aM. Andresen, M. C. Wahl, A. C. Stiel, F. Grater, L. V. Schafer, S. Trowitzsch, G. Weber, C. Eggeling, H. Grubmuller, S. W. Hell, S. Jakobs, Proceedings of the National Academy of Sciences of the United States of America 2005, 102, 13070-13074; bM. Andresen, A. C. Stiel, S. Trowitzsch, G. Weber, C. Eggeling, M. C. Wahl, S. W. Hell, S. Jakobs, Proceedings of the National Academy of Sciences of the United States of America 2007, 104, 13005-13009.

[10] N. A. Jensen, I. Jansen, M. Kamper, S. Jakobs, in Nanoscale Photonic Imaging (Eds.: T. Salditt, A. Egner, D. R. Luke), Springer International Publishing, Cham, 2020, pp. 241-261.

[11] H. Shinoda, K. Lu, R. Nakashima, T. Wazawa, K. Noguchi, T. Matsuda, T. Nagai, Cell Chem Biol 2019, 26, 1469-1479 e1466.

[12] D. Ruhlandt, M. Andresen, N. Jensen, I. Gregor, S. Jakobs, J. Enderlein, A. I. Chizhik, Communications Biology 2020, 3, 627.

[13] X. Zhang, M. Zhang, D. Li, W. He, J. Peng, E. Betzig, P. Xu, Proceedings of the National Academy of Sciences of the United States of America 2016, 113, 10364-10369.

[14] N. E. Christou, K. Giandoreggio-Barranco, I. Ayala, O. Glushonkov, V. Adam, D. Bourgeois, B. Brutscher, J Am Chem Soc 2021, 143, 7521-7530.

[15] aE. De Zitter, D. Thédié, V. Mönkemöller, S. Hugelier, J. Beaudouin, V. Adam, M. Byrdin, L. Van Meervelt, P. Dedecker, D. Bourgeois, Nature Methods 2019, 16, 707710; bE. De Zitter, J. Ridard, D. Thédié, V. Adam, B. Lévy, M. Byrdin, G. Gotthard, L. 
Van Meervelt, P. Dedecker, I. Demachy, D. Bourgeois, Journal of the American Chemical Society 2020, 142, 10978-10988.

[16] D. Morozov, G. Groenhof, Angew Chem Int Ed Engl 2016, 55, 576-578.

[17] T. Grotjohann, I. Testa, M. Reuss, T. Brakemann, C. Eggeling, S. W. Hell, S. Jakobs, eLife 2012, 1, e00248.

[18] J. Woodhouse, G. Nass Kovacs, N. Coquelle, L. M. Uriarte, V. Adam, T. R. M. Barends, M. Byrdin, E. de la Mora, R. Bruce Doak, M. Feliks, M. Field, F. Fieschi, V. Guillon, S. Jakobs, Y. Joti, P. Macheboeuf, K. Motomura, K. Nass, S. Owada, C. M. Roome, C. Ruckebusch, G. Schirò, R. L. Shoeman, M. Thepaut, T. Togashi, K. Tono, M. Yabashi, M. Cammarata, L. Foucar, D. Bourgeois, M. Sliwa, J.-P. Colletier, I. Schlichting, M. Weik, Nature Communications 2020, 11, 741.

[19] J. Chang, M. G. Romei, S. G. Boxer, J Am Chem Soc 2019, 141, 15504-15508.

[20] N. Coquelle, M. Sliwa, J. Woodhouse, G. Schirò, V. Adam, A. Aquila, T. R. M. Barends, S. Boutet, M. Byrdin, S. Carbajo, E. De la Mora, R. B. Doak, M. Feliks, F. Fieschi, L. Foucar, V. Guillon, M. Hilpert, M. S. Hunter, S. Jakobs, J. E. Koglin, G. Kovacsova, T. J. Lane, B. Lévy, M. Liang, K. Nass, J. Ridard, J. S. Robinson, C. M. Roome, C. Ruckebusch, M. Seaberg, M. Thepaut, M. Cammarata, I. Demachy, M. Field, R. L. Shoeman, D. Bourgeois, J.-P. Colletier, I. Schlichting, M. Weik, Nature Chemistry 2018, 10, 31-37.

[21] M. El Khatib, A. Martins, D. Bourgeois, J. P. Colletier, V. Adam, Scientific reports 2016, 6, 18459.

[22] G. Schiro, J. Woodhouse, M. Weik, I. Schlichting, R. L. Shoeman, Journal of Applied Crystallography 2017, 50, 932-939.

[23] W. W. Ward, Vol. xxviii (Ed.: M. A. D. a. W. D. McElroy), New York, Academic Press, 1981, p. 782.

[24] S. Duwé, E. De Zitter, V. Gielen, B. Moeyaert, W. Vandenberg, T. Grotjohann, K. Clays, S. Jakobs, L. Van Meervelt, P. Dedecker, ACS nano 2015, 9, 9528-9541.

[25] J. Langeland, N. W. Persen, E. Gruber, H. V. Kiefer, A. M. Kabylda, A. V. Bochenkova, L. H. Andersen, Chemphyschem 2021, 22, 833-841.

[26] S. Schnorrenberg, T. Grotjohann, G. Vorbrüggen, A. Herzig, S. W. Hell, S. Jakobs, eLife 2016, 5, e15567.

[27] H. Frauenfelder, S. G. Sligar, P. G. Wolynes, Science 1991, 254, 1598-1603. 